An audit into the use of psychotropic medications in patients with autism spectrum disorder in a high security psychiatric hospital

Elliott Carthy* and David Murphy

Broadmoor Hospital, West London NHS Trust

${ }^{\star}$ Corresponding author.

doi: 10.1192/bjo.2021.222

Aims. Autism Spectrum Disorder (ASD) is a common neurodevelopmental disorder associated with difficulties in social communication and language development, preoccupations, a need for routine, sensory sensitivities and emotional dysregulation. People with autism who have violently offended may be prescribed psychotropic medications to treat comorbidities, or offlicense to manage aggressive or challenging behaviours. However, the evidence base for their use remains scarce.

Method. This was a retrospective audit at Broadmoor Hospital, a high security psychiatric hospital in the United Kingdom, into the safe and appropriate prescribing of psychotropic medicines in those with an ASD compared to guidance from the National Institute for Health and Care Excellence (CG142): "Autism spectrum disorder in adults: diagnosis and management". This first cycle was undertaken during May and June 2020 and included all patients with a confirmed or equivocal diagnosis of ASD in the preceding five years.

Result. A total of 22 participants were included in this study. Of these, 17 participants had a confirmed diagnosis of ASD and five participants had a suspected diagnosis of ASD, but without formal confirmation with neurocognitive testing. A total of 13 (76.5\%) participants with confirmed ASD were prescribed antipsychotic medication, nine of whom had an established comorbid mental disorder with psychotic symptoms. Of the remaining four, three had a diagnosis of a personality disorder. Three participants in this study had a confirmed diagnosis of ASD without any additional comorbid mental health diagnoses. No patients were prescribed psychotropic medicines for the core symptoms of ASD. The specific documentation of off-license use of antipsychotic medicines in those without a diagnosis of a psychotic disorder was poor. This was not recorded in any such participant in the preceding 12 months.

Conclusion. This audit highlighted that dual diagnoses of ASD alongside non-affective psychosis and personality disorder are overrepresented in this high security setting. The NICE clinical guidelines CG142 guidelines state that "antipsychotic medications should only be used for behaviour that challenges if .... the risk to the person or others is very severe". By definition, all patients admitted to high security are deemed to be a grave and imminent risk to the public. Psychotropic medicines may therefore be clinically indicated at a much earlier stage than in community patients, instigated alongside appropriate psychosocial interventions and treatment of comorbid conditions. It may be that catered guidelines need to be formulated to support the safe and appropriate prescribing of psychotropic medicine in forensic settings.

\section{An audit into the management of diabetes mellitus at Broadmoor Hospital \\ Elliott Carthy ${ }^{1 \star}$ and Callum Ross ${ }^{2}$ \\ ${ }^{1}$ Oxford Health NHS Foundation Trust and ${ }^{2}$ Broadmoor Hospital, Crowthorne Berks \\ ${ }^{\star}$ Corresponding author.}

doi: 10.1192/bjo.2021.223
Aims. Diabetes mellitus confers a twofold excess risk of cardiovascular disease - the leading cause of premature mortality in those with severe mental illness. Inpatients in forensic settings often have more severe, enduring and treatment-resistance forms of mental illness, sometimes necessitating combinations of prescribed antipsychotics. This audit aimed to assess adherence to National Institute for Health and Care Excellence (NICE) guidelines NG28 titled "type 2 diabetes in adults: management" and summarise the metabolic parameters of those with diabetes mellitus at Broadmoor hospital.

Method. This was a retrospective audit in a high secure forensic psychiatry hospital in the United Kingdom, into the management of patients with diabetes mellitus compared to guidance from NICE (NG28).

Result. We report data from over 30 inpatients (out of approximately 200) at a high secure forensic psychiatry hospital with a diagnosis of type 2 diabetes mellitus across two audit cycles. This audit identified improved adherence to national guidance regarding six monthly monitoring of $\mathrm{HbAlc}$ but with less than $50 \%$ of such patients having an HbAlc at or below the recommended target. This is in addition to high rates of other metabolic disorders such as obesity, dyslipidaemia and hypertension and a mean QRISK3 score that was markedly higher than a healthy person with the same age, sex, and ethnicity. There was a prevalence of background diabetic retinopathy of $8 \%$, diabetic nephropathy of $5.4 \%$, no recorded cases of diabetic neuropathy and a macrovascular disease prevalence of $5.4 \%$. There were no new diagnoses of microvascular or macrovascular disease between audit cycles. One of the key changes between audit cycles was the recruitment of a dietician to the hospital. By the time of undertaking the second audit cycle, 23 patients had documented evidence of having been offered a referral to the dietician.

Conclusion. This audit highlighted the marked cardiovascular risk in patients with type 2 diabetes mellitus at a high secure forensic psychiatry hospital. This includes suboptimal control of blood pressure, lipid profiles and HbAlc that increases the risk of premature mortality in these patients with severe mental illness. Wider, cultural changes in practice need to be implemented to improve the metabolic health of patients in the long-term inpatient setting of Broadmoor Hospital. This includes prescribers avoiding the most diabetogenic antipsychotics where possible, increasing the provision of sugar-free options at the on-site shop, examining the proportion of carbohydrate-rich foodstuffs in the shop and understanding the characteristics of its heaviest purchasers, and continued coordination between primary care and ward teams to support patients in making sustained changes to improve their metabolic health.

\section{An audit into the management of COVID-19 in a high security psychiatric hospital}

Elliott Carthy ${ }^{1 \star}$ and Samrat Sengupta ${ }^{2}$

${ }^{1}$ Warneford Hospital and ${ }^{2}$ Broadmoor Hospital, Crowthorne Berks ${ }^{*}$ Corresponding author.

doi: 10.1192/bjo.2021.224

Aims. Comprehensive and timely data collection during a pandemic is crucial in developing guidelines and policy as well as evaluating their effectiveness. In turn, this will improve planning for future incidents. While this is being undertaken at a national level by Public Health England, more specific information as it relates to psychiatric care is important in understanding the neuropsychiatric, psychological and social effects of the pandemic. The management of patients with COVID-19 presents a 
unique challenge in inpatient psychiatry settings both in terms of diagnosis and treatment. This is perhaps greater still in forensic settings due to the increased risk of violence and aggression. This audit aimed to firstly assess the consistency of local practice to national guidance from Public Health England. Secondly, it aimed to describe the clinical management of suspected and confirmed cases of COVID-19 in this high security forensic hospital and how readily broad, national guidance can be implemented in this unique setting. We present an audit with three cycles, one from each wave of COVID-19 in England during 2020.

Method. This was a retrospective audit in a high secure forensic psychiatry hospital in the United Kingdom, into the investigation and management of suspected and confirmed cases of COVID-19 compared to national guidelines from Public Health England. It includes three cycles, one undertaken in each national wave of COVID-19 in England in 2020.

Result. Ten patients have been included in cycle 1, 12 in cycle 2 and 21 in cycle 3 as those where COVID-19 was a considered diagnosis. SARS-CoV-2 was detected in one patient in cycle 1 and 12 patients in cycle 3. All patients recovered, most of whom remained on-site with supportive care in self-isolation on a dedicated ward for positive cases. Three patients required additional treated with oral antibiotics and dexamethasone, one of whom required admission to the local general medical hospital for continuous supplemental oxygen.

Conclusion. This is the first study to describe the management of the novel COVID-19 pandemic in a high security forensic psychiatry hospital and how readily national guidance can be implemented in this unique setting. Hospital practice at identifying suspected cases and the management of confirmed cases of COVID-19 was shown to be consistent with national guidance. It also allowed for clinicians to exercise their judgement about testing for atypical cases and for repeat testing where appropriate.

Audit examining memantine initiation in dementia patients in an older adult service in the north west

Irina Casapu ${ }^{1 *}$, Ste Dickinson ${ }^{2}$, Chirag Shroff ${ }^{3}$, Sofia Almeida ${ }^{4}$ and Kieran McSharry

${ }^{1}$ CT3, Liaison Psychiatry, Aintree Hospital; ${ }^{2}$ F3, A and E, Royal Liverpool University Hospital; ${ }^{3}$ CT2, Burlington House CAMHS, Alderhey NHS Trust; ${ }^{4}$ CT3, Liaison Psychiatry, Countess of Chester Hospital and ${ }^{5}$ ST5 Dual Old Age and General Adult Trainee, Merseycare NHS

${ }^{*}$ Corresponding author.

doi: 10.1192/bjo.2021.225

Aims. Dementia is a progressive condition inflicting significant costs for health and social care services. In December 2017, there were 456,739 people on GP registers with a formal diagnosis of dementia. Making the right choice of anti-dementia medication with essential monitoring is one important aspect of care. Thus, the aim of this audit was to identify if current practice at Mossley Hill inpatients and outpatients service for older adults in Liverpool, was in accordance with the NICE Guideline NG97 (Dementia: assessment, management and support for people living with dementia and their carers). Additionally, we aimed to evaluate whether Memantine was commenced according to $\mathrm{BNF} / \mathrm{SPC}$ recommendations about e-GFR and whether this was documented on patient records, as well as to highlight areas of improvement.

Method. An audit was carried out for all patients for whom Memantine was initiated, between June and August 2019. Sixty-nine patients were identified through trust Pharmacy records. Data were collected retrospectively, reviewing local electronic records (ePEX, RIO) and GP referrals. This included age, sex, diagnosis, indication for starting Memantine, decision context, prescriber, documentation of renal function status and communication of decision to the GP. Findings were compared to NICE guidance NG97 and presented at the local audit meeting with a view to recommend strategies for improvement.

Result. Results indicated that most of the patients were female (64\%) with the most common diagnosis being Alzheimer's disease (75\%). Recurrent reasons for initiating Memantine were: contraindication for AChE treatment (25\%); illness progression on AChE (22\%); and severe dementia on initial presentation (23\%). Usually, the decision to start Memantine treatment was made in MDT or after prescriber clinical review. In $68 \%$ of the reviewed cases, renal function status was documented. Patients' GP was informed of medication change in $86 \%$ of cases.

Conclusion. To conclude, in the majority of cases Memantine initiation was in line with NICE guidance. However, documentation can be improved, so as to facilitate future audit. We recommended creating a checklist for prescribing Memantine that could be integrated within the electronic records system.

\section{Transition from Methylphenidate to Atomoxetine: reasons for switching and clinical outcome}

Fabrizia Cassar ${ }^{\star}$, Giovanni Grech, Bertha Grech and Joseph Cassar

Mount Carmel Hospital

${ }^{\star}$ Corresponding author.

doi: 10.1192/bjo.2021.226

Aims. Attention Deficit Hyperactivity Disorder (ADHD) is a behavior disorder originating in childhood comprising of a constellation of features including inattention, impulsivity, and hyperactivity. The National Institute of Clinical Excellence (NICE) Guidelines 2018 recommends methylphenidate as a first line pharmacological agent for treatment of children aged 5 years and over with ADHD. Lisdexamfetamine, dexamfetamine and atomoxetine are recommended in this order if methylphenidate is not tolerated or if symptoms did not respond to separate 6-week trials. Our aim was to, assess the transition of methylphenidate to atomoxetine, the reasons for switching and its clinical outcome in order to make recommendations to current practice regarding treatment of ADHD.

Method. The study examined a total of 53 children between 0-16 years of age who were being treated for ADHD with atomoxetine at CYPS till September 2018. Data was collected from patients' files retrospectively by using a proforma based on the NICE guidelines 2018 ADHD: diagnosis and management.

Result. Out of 53 patients' on atomoxetine in September 2018, 49 were included in the study. Results recorded side-effects as the main reason for switching from methylphenidate to atomoxetine. Unwanted side-effects were documented in $71.7 \%$ of patients of which $57.9 \%$ exhibited more than 1 side-effect with the two commonest side-effects documented being weight loss and decreased appetite. The audit highlighted the fact that the correct dose of atomoxetine was only administered in $17.2 \%$ of children with $56.9 \%$ of patient's being given a higher dose than recommended. Initial weight was not documented in $19 \%$ and hence, ideal dose could not be calculated. Overall, atomoxetine was shown to be an effective treatment. Out of the 40 patients documented to have hyperactivity this symptom was decreased in $82.5 \%$ whilst $82.9 \%$ were shown to have increased concentration. 35 patients had documented impulsivity and this was decreased in $62.9 \%$ of 